\title{
MHD Flow and Heat Transfer in a Channel Bounded by a Shrinking Sheet and a Porous Medium Bed: Homotopy Analysis Method
}

\author{
Dileep Singh Chauhan and Rashmi Agrawal \\ Department of Mathematics, University of Rajasthan, Jaipur 302004, India \\ Correspondence should be addressed to Dileep Singh Chauhan; dileepschauhan@gmail.com
}

Received 26 October 2012; Accepted 15 November 2012

Academic Editors: G. L. Aranovich and R. R. Burnette

Copyright ( 2013 D. S. Chauhan and R. Agrawal. This is an open access article distributed under the Creative Commons Attribution License, which permits unrestricted use, distribution, and reproduction in any medium, provided the original work is properly cited.

\begin{abstract}
MHD flow of viscous conducting fluid is considered between a shrinking sheet and a porous medium bed. Suction is applied at the upper shrinking sheet and its surface temperature is always maintained higher than the temperature of the lower porous bed surface. Similarity transformations and HAM are used to solve the governing equations for velocity and temperature fields. The effects of various pertinent parameters on the results are discussed graphically.
\end{abstract}

\section{Introduction}

The study of boundary layer viscous flow and heat transfer due to stretching/shrinking surfaces is very significant because of its several applications in engineering and industrial processes, such as extrusion of polymer sheets from a die, drawing of plastic films, polyester thin wall heat shrink tubing, shrink film, wire drawing, glass fiber, and paper production. It is very important to control drag and the rate of heat transfer from the sheet for a better quality of the final product. Crane [1] first examined such flow over a linearly stretching sheet and obtained an exact similarity solution for the steady boundary layer flow of a viscous fluid in a closed form. His work was extended under various situations and physical conditions, by several researchers, such as Rajagopal et al. [2], Dandapat and Gupta [3], Chiam [4], Cortell [5], Liu [6], Ariel et al. [7, 8], Abbas et al. [9], Abd El-Aziz [10], Chauhan and Rastogi [11], Chauhan and Agrawal [12], and Chauhan and Olkha [13].

Recently the study of boundary layer flow and heat transfer adjacent to a shrinking sheet is given attention because of its several industrial applications, particularly in packaging industry. Flow due to a shrinking sheet is relatively a new phenomenon, where the velocity on the sheet is directed towards a fixed point. Steady flow in such a case does not occur normally, since the vorticity generated due to the shrinking of the sheet is not confined in the boundary layer unless a proper suction is applied at the sheet. Wang [14] reported the flow development adjacent to a shrinking sheet. Miklavčič and Wang [15] studied the existence and uniqueness of steady flow due to shrinking sheet and reported that for specific suction values dual solutions exist. Wang [16] studied stagnation-point flow over a shrinking sheet. Nadeem and Awais [17] investigated fluid flow through porous medium due to unsteady shrinking sheet with variable viscosity. Fang et al. [18] obtained a new solution for the Blasius equation of a shrinking sheet problem. Muhaimin et al. [19] investigated effects of heat and mass transfer on MHD flow over a shrinking sheet with suction. Rahimpour et al. [20] obtained the analytical solution of axisymmetric stagnation flow towards a shrinking sheet. Using HAM, Hayat et al. [21] investigated MHD rotating flow over a shrinking surface. Sajid and Hayat [22] examined MHD viscous flow due to a shrinking sheet using homotopy analysis method. The boundary layer flow adjacent to a shrinking sheet with power-law surface velocity is investigated by Fang [23] with mass transfer. A closed form analytical solution was presented by Fang and Zhang [24] for MHD flow over a shrinking sheet. Fang et al. [25] investigated the unsteady flow over a shrinking sheet with mass suction. 
Yao and Chen [26] presented the new analytical solution of Blasius equation for a shrinking sheet problem. Mohd and Hashim [27] studied MHD flow and heat transfer over a shrinking sheet embedded in a porous medium with suction. Cortell [28] examined MHD flow due to shrinking sheet for axisymmetric and two-dimensional cases with suction. Noor et al. [29] obtained a series solution for MHD flow due to shrinking sheet by ADM (Adomian decomposition method). Hayat et al. [30] developed an analytical solution for a rotating flow due to a shrinking sheet. Fang and Zhang [31] presented an exact analytical solution for thermal boundary layer over a shrinking sheet with mass transfer effects. Fang et al. [32] investigated analytically flow adjacent to a shrinkingsheet with a second-order slip flow model. Ishak et al. [33] investigated stagnation-point flow of a micropolar fluid over a shrinking sheet. The channel flows with stretching/shrinking walls are also very important problems in fluid dynamics. Brady and Acrivos [34], Borkakoti and Bharali [35], Chauhan and Jakhar [36], Hayat et al. [37], and Chauhan and Agrawal [38] investigated flow and heat transfer problem in channels with one wall as stretching or shrinking sheet.

The purpose of the present investigation is to discuss the analytically solutions of electrically conducting viscous fluid flow and heat transfer in a channel bounded by a porous shrinking sheet and a porous medium bed of very small permeability, in the presence of a magnetic field. The equations governing velocity and temperature fields are simplified by a suitable similarity transformation, and then the reduced nonliner boundary value problem is solved analytically using homotopy analysis method. The physical effects of pertinent parameters on the flow and heat transfer are depicted graphically and discussed. The convergence of the solutions has also discussed by the so-called h-curves.

\section{Formulation of the Problem}

An electrically conducting, incompressible, viscous fluid flow in a horizontal channel bounded by a shrinking sheet and a porous medium bed is considered. A constant suction $w_{0}$ is applied at the upper shrinking sheet of the channel. The lower porous bed of the channel is of very small permeability $k_{0}$. We model flow in porous bed by Darcy's law. So, the flow in the porous bed is assumed to be zero in the absence of any external pressure gradient. A slip boundary condition suggested by Saffman [39] is applied at the fluid-porous medium interface. The permeability of the porous bed affects the flow in the channel through this boundary condition. Thus the effect of the porous bed is to introduce slip at the lower boundary of the horizontal channel. A constant magnetic field of strength $B_{0}$ is applied to the normal direction of the horizontal channel. Magnetic Reynolds number is taken to be small; therefore, induced magnetic field is neglected. No external electric field is applied and the polarization effect of the ionized fluid is negligible. So the electric field $E$ is assumed to be zero. A Cartesian coordinate system $(x, y, z)$ is assumed with origin at the porous bed surface. The axis of $x$ and axis of $y$ are taken on the surface of the porous bed and the axis of $z$ is normal to it (see schematic diagram, Figure 1).

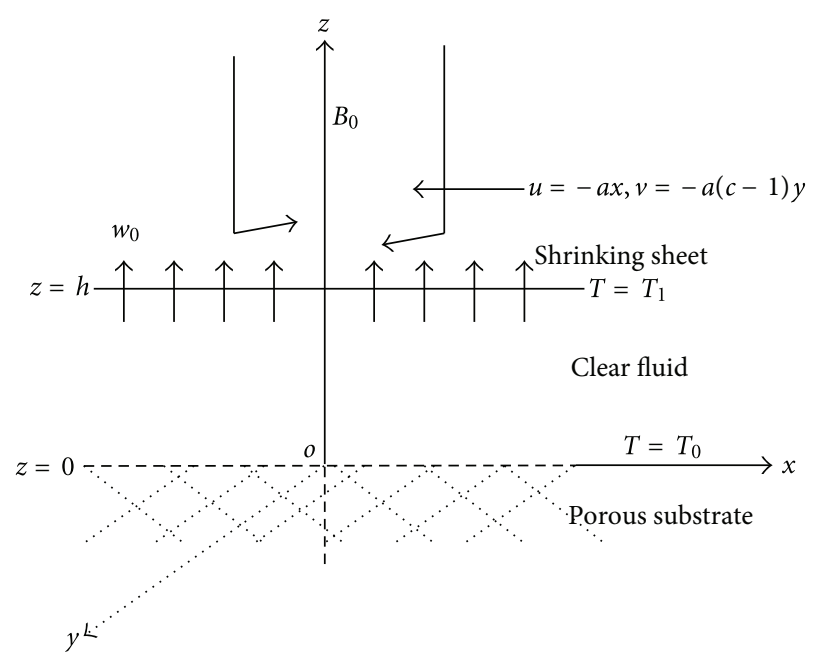

FIGURE 1: Schematic diagram.

$(u, v, w)$ are considered velocity components in $x, y$, and $z$ directions, respectively, and $T$ is the temperature of the fluid in the channel. The width of the channel is assumed $h$, so that walls of the channel are at $z=0$ and $z=h$.

The governing equations for the present problem of MHD flow and heat transfer are given by

$$
\begin{gathered}
\frac{\partial u}{\partial x}+\frac{\partial v}{\partial y}+\frac{\partial w}{\partial z}=0 \\
u \frac{\partial u}{\partial x}+v \frac{\partial u}{\partial y}+w \frac{\partial u}{\partial z}=-\frac{1}{\rho} \frac{\partial P}{\partial x}+v\left(\frac{\partial^{2} u}{\partial x^{2}}+\frac{\partial^{2} u}{\partial y^{2}}+\frac{\partial^{2} u}{\partial z^{2}}\right)-\frac{\sigma B_{0}^{2}}{\rho} u \\
u \frac{\partial v}{\partial x}+v \frac{\partial v}{\partial y}+w \frac{\partial v}{\partial z}=-\frac{1}{\rho} \frac{\partial P}{\partial y}+v\left(\frac{\partial^{2} v}{\partial x^{2}}+\frac{\partial^{2} v}{\partial y^{2}}+\frac{\partial^{2} v}{\partial z^{2}}\right)-\frac{\sigma B_{0}^{2}}{\rho} v \\
u \frac{\partial w}{\partial x}+v \frac{\partial w}{\partial y}+w \frac{\partial w}{\partial z}=-\frac{1}{\rho} \frac{\partial P}{\partial z}+v\left(\frac{\partial^{2} w}{\partial x^{2}}+\frac{\partial^{2} w}{\partial y^{2}}+\frac{\partial^{2} w}{\partial z^{2}}\right) \\
\rho C_{p}\left(u \frac{\partial T}{\partial x}+v \frac{\partial T}{\partial y}+w \frac{\partial T}{\partial z}\right)=k\left(\frac{\partial^{2} T}{\partial x^{2}}+\frac{\partial^{2} T}{\partial y^{2}}+\frac{\partial^{2} T}{\partial z^{2}}\right)
\end{gathered}
$$

The corresponding boundary conditions are given by

$$
\begin{gathered}
\text { at } z=h, \quad u=-a x, \quad v=-a(c-1) y, \\
w=w_{0}, \quad T=T_{1}, \\
\text { at } z=0, \quad \tau_{z x}=\frac{\mu \alpha}{\sqrt{k_{0}}} u, \quad \tau_{z y}=\frac{\mu \alpha}{\sqrt{k_{0}}} v, \\
w=0, \quad T=T_{0},
\end{gathered}
$$

where $P$ is the pressure; $\rho$ is the density of the fluid; $v$ is the kinematic viscosity; $\sigma$ is the electrical conductivity; $C_{p}$ 
is the specific heat at constant pressure; $k$ is the thermal conductivity in the clear fluid medium; $T_{0}$ is the porous medium wall temperature; $T_{1}$ is the temperature of upper stretching wall; $k_{0}$ is the permeability of the porous medium; $\mu$ is the viscosity of the clear fluid; $\alpha$ is a nondimensional constant that depends only on the structure of the porous matrix.

Here, $a>0$ is the shrinking constant. $c$ is a constant, where $c=1$ indicates one-direction shrinking and $c=2$ corresponds to axisymmetric shrinking.

\section{Method of Solution}

We introduce the following similarity transformations:

$$
\begin{gathered}
u=\operatorname{ax} f^{\prime}(\eta), \quad v=a(c-1) y f^{\prime}(\eta), \quad w=-\operatorname{ahc} f(\eta), \\
\eta=\frac{z}{h}, \quad \theta(\eta)=\frac{T-T_{0}}{T_{1}-T_{0}},
\end{gathered}
$$

where $T_{1}-T_{0}=D x, D$ being a nonzero constant.

By using similarity transformations defined in (7), we find that the continuity equation (1) is automatically satisfied. Further, we see that (4) reduces to

$$
w \frac{\partial w}{\partial z}=-\frac{1}{\rho} \frac{\partial P}{\partial z}+v\left(\frac{\partial^{2} w}{\partial z^{2}}\right) .
$$

On integration, we get

$$
\frac{w^{2}}{2}=-\frac{P}{\rho}+v \frac{d w}{d z}+\text { constant }
$$

Equations (2) and (3), using (9) and similarity transformations (7), are reduced to

$$
f^{i v}-M^{2} f^{\prime \prime}-R\left((2-c) f^{\prime} f^{\prime \prime}-c f f^{\prime \prime \prime}\right)=0 .
$$

Similarly, using (7), we see that (5) is reduced to

$$
\theta^{\prime \prime}=R \operatorname{Pr}\left(f^{\prime} \theta-c f \theta^{\prime}\right)
$$

The corresponding boundary conditions for the present problem are reduced to

$$
\begin{gathered}
\text { at } \eta=1, \quad f^{\prime}=-1, \quad f=-\frac{w_{0}}{\mathrm{ach}}=-\lambda, \quad \theta=1, \\
\text { at } \quad \eta=0, \quad f^{\prime \prime}=\frac{\alpha}{K} f^{\prime}, \quad f=0, \quad \theta=0,
\end{gathered}
$$

where $M=\sqrt{(\sigma / \rho v)} B_{0} h$, the Hartmann number; $R=a h^{2} / v$, the nondimensional shrinking parameter; $\operatorname{Pr}=\mu C_{p} / k$, the Prandtl number; $K=\sqrt{k_{0}} / h$, the permeability parameter.

\section{Homotopy Analysis Method}

Following Liao [40], we now solve the nonlinear system consisting of (10)-(12) by HAM. For HAM solutions, we choose the initial guesses $f_{0}(\eta)$ and $\theta_{0}(\eta)$ and the auxiliary linear operators $L_{f}$ and $L_{\theta}$ in the following forms:

$$
\begin{gathered}
f_{0}(\eta)=\frac{2 K(1-3 \lambda)}{4 K+\alpha} \eta+\frac{\alpha(1-3 \lambda)}{4 K+\alpha} \eta^{2} \\
+\frac{\alpha(2 \lambda-1)-2 K(1-\lambda)}{4 K+\alpha} \eta^{3}, \\
\theta_{0}(\eta)=\eta, \\
L_{f}(f)=\frac{d^{4} f}{d \eta^{4}}, \\
L_{\theta}(\theta)=\frac{d^{2} \theta}{d \eta^{2}} .
\end{gathered}
$$

Here, auxiliary linear operators have the property that

$$
\begin{gathered}
L_{f}\left(f_{0}\right)=L_{f}\left(a_{0,0}+a_{0,1} \eta+a_{0,2} \eta^{2}+a_{0,3} \eta^{3}\right)=0, \\
L_{\theta}\left(\theta_{0}\right)=L_{\theta}\left(b_{0,0}+b_{0,1} \eta\right)=0 .
\end{gathered}
$$

Let $p \in[0,1]$ denote the embedding parameter and $\mathrm{h}_{1}, \mathrm{~h}_{2}$ indicate the nonzero auxiliary parameters. Then we construct the following homotopies:

$$
\begin{aligned}
H_{f}[\bar{f}(\eta ; p) ; p]= & (1-p) L_{f}\left[\bar{f}(\eta ; p)-f_{0}(\eta)\right] \\
& -p \hbar_{1} N_{f}[\bar{f}(\eta ; p)], \\
H_{\theta}[\bar{\theta}(\eta ; p) ; p]= & (1-p) L_{\theta}\left[\bar{\theta}(\eta ; p)-\theta_{0}(\eta)\right] \\
& -p \hbar_{2} N_{\theta}[\bar{\theta}(\eta ; p)],
\end{aligned}
$$

where

$$
\begin{aligned}
N_{f}[\bar{f}(\eta ; p)]= & \frac{\partial^{4} \bar{f}(\eta ; p)}{\partial \eta^{4}}-M^{2} \frac{\partial^{2} \bar{f}(\eta ; p)}{\partial \eta^{2}} \\
& -R\left[(2-c) \frac{\partial \bar{f}(\eta ; p)}{\partial \eta} \frac{\partial^{2} \bar{f}(\eta ; p)}{\partial \eta^{2}}\right. \\
\left.-c \bar{f}(\eta ; p) \frac{\partial^{3} \bar{f}(\eta ; p)}{\partial \eta^{3}}\right], & \\
N_{\theta}[\bar{\theta}(\eta ; p)]= & \frac{\partial^{2} \bar{\theta}(\eta ; p)}{\partial \eta^{2}}-R \operatorname{Pr} \\
& \times\left[\bar{\theta}(\eta ; p) \frac{\partial \bar{f}(\eta ; p)}{\partial \eta}-c \bar{f}(\eta ; p) \frac{\partial \bar{\theta}(\eta ; p)}{\partial \eta}\right] .
\end{aligned}
$$

When $p=0$, we have

$$
\begin{aligned}
H_{f}[\bar{f}(\eta ; 0) ; 0] & =L_{f}\left[\bar{f}(\eta ; 0)-f_{0}(\eta)\right] \\
H_{\theta}[\bar{\theta}(\eta ; 0) ; 0] & =L_{\theta}\left[\bar{\theta}(\eta ; 0)-\theta_{0}(\eta)\right] .
\end{aligned}
$$


If $p=1$, we have

$$
\begin{aligned}
& H_{f}[\bar{f}(\eta ; 1) ; 1]=-\hbar_{1} N_{f}[\bar{f}(\eta ; 1)] \\
& H_{\theta}[\bar{\theta}(\eta ; 1) ; 1]=-\hbar_{2} N_{\theta}[\bar{\theta}(\eta ; 1)] .
\end{aligned}
$$

4.1. Zeroth Order Deformation Equations. Thus, if we take

$$
\begin{aligned}
& H_{f}[\bar{f}(\eta ; p) ; p]=0, \\
& H_{\theta}[\bar{\theta}(\eta ; p) ; p]=0,
\end{aligned}
$$

we obtain the family of equations as follows:

$$
\begin{gathered}
(1-p) L_{f}\left[\bar{f}(\eta ; p)-f_{0}(\eta)\right]=p \hbar_{1} N_{f}[\bar{f}(\eta ; p)] \\
(1-p) L_{\theta}\left[\bar{\theta}(\eta ; p)-\theta_{0}(\eta)\right]=p \hbar_{2} N_{\theta}[\bar{\theta}(\eta ; p), \bar{f}(\eta ; p)] .
\end{gathered}
$$

These equations are subjected to the boundary conditions:

$$
\begin{gathered}
\overline{f^{\prime}}(1 ; p)=-1, \quad \bar{f}(1 ; p)=-\lambda, \quad \bar{\theta}(1 ; p)=1, \\
\overline{f^{\prime \prime}}(0 ; p)=\frac{\alpha}{K} \overline{f^{\prime}}(0 ; p), \quad \bar{f}(0 ; p)=0, \quad \bar{\theta}(0 ; p)=0 .
\end{gathered}
$$

Obviously, when $p=0$, we see that

$$
\begin{aligned}
& \bar{f}(\eta ; 0)=f_{0}(\eta), \\
& \bar{\theta}(\eta ; 0)=\theta_{0}(\eta),
\end{aligned}
$$

and when $p=1$, we have

$$
\begin{aligned}
& \bar{f}(\eta ; 1)=f(\eta), \\
& \bar{\theta}(\eta ; 1)=\theta(\eta) .
\end{aligned}
$$

Thus, as the embedding parameter $p$ increases from 0 to 1 , the mappings $\bar{f}(\eta ; p)$ and $\bar{\theta}(\eta ; p)$ vary smoothly from their known initial guesses, $f_{0}(\eta)$ and $\theta_{0}(\eta)$, to the required solutions, $f(\eta)$ and $\theta(\eta)$, respectively.

Expanding $\bar{f}(\eta ; p)$ and $\bar{\theta}(\eta ; p)$ by Taylor's series in the embedding parameter $p$ and using (22), we can write

$$
\bar{f}(\eta ; p)=f_{0}(\eta)+\sum_{k=1}^{\infty} f_{k}(\eta) p^{k}
$$

where $f_{k}(\eta)=\left[(1 / k !)\left(\partial^{k} \bar{f}(\eta ; p) / \partial p^{k}\right)\right]_{p=0}$ and

$$
\bar{\theta}(\eta ; p)=\theta_{0}(\eta)+\sum_{k=1}^{\infty} \theta_{k}(\eta) p^{k}
$$

where $\theta_{k}(\eta)=\left[(1 / k !)\left(\partial^{k} \bar{\theta}(\eta ; p) / \partial p^{k}\right)\right]_{p=0}$.
Now assume that for properly chosen values of $\hbar_{1}$ and $\hbar_{2}$ the series (24) and the series (25) are convergent at $p=1$. Thus we obtain

$$
\begin{aligned}
& f(\eta)=f_{0}(\eta)+\sum_{k=1}^{\infty} f_{k}(\eta), \\
& \theta(\eta)=\theta_{0}(\eta)+\sum_{k=1}^{\infty} \theta_{k}(\eta) .
\end{aligned}
$$

4.2. $m$ th Order Deformation Equations. The governing equations of $f_{m}(\eta)$ and $\theta_{m}(\eta)$ can be deduced from the zeroth order deformation equations (20) by differentiating these $m$ times with respect to the embedding parameter $p$, setting $p=0$ and finally dividing them by $\mid \underline{m}$. Thus we have

$$
\begin{aligned}
& L_{f}\left[f_{m}(\eta)-\chi_{m} f_{m-1}(\eta)\right]=\hbar_{1} R_{m}^{f}(\eta), \\
& L_{\theta}\left[\theta_{m}(\eta)-\chi_{m} \theta_{m-1}(\eta)\right]=\hbar_{2} R_{m}^{\theta}(\eta),
\end{aligned}
$$

subject to corresponding boundary conditions:

$$
\begin{gathered}
f_{m}^{\prime}(1)=0, \quad f_{m}(1)=0, \quad \theta_{m}(1)=0, \\
f_{m}^{\prime \prime}(0)=\frac{\alpha}{K} f_{m}^{\prime}(0), \quad f_{m}(0)=0, \quad \theta_{m}(0)=0,
\end{gathered}
$$

where

$$
\begin{aligned}
R_{m}^{f}(\eta)= & \left.\frac{1}{\underline{\underline{m-1}}} \frac{\partial^{m-1} N_{f}[\bar{f}(\eta ; p)]}{\partial p^{m-1}}\right|_{p=0} \\
= & f_{m-1}^{i v}-M^{2} f_{m-1}^{\prime \prime} \\
& +\operatorname{Re} \sum_{r=0}^{m-1}\left((2-c) f_{m-1-r}^{\prime} f_{r}^{\prime \prime}-c f_{m-1-r} f_{r}^{\prime \prime \prime}\right), \\
R_{m}^{\theta}(\eta)= & \left.\frac{1}{\mid m-1} \frac{\partial^{m-1} N_{\theta}[\bar{\theta}(\eta ; p), \bar{f}(\eta ; p)]}{\partial p^{m-1}}\right|_{p=0} \\
= & \theta_{m-1}^{\prime \prime}-R \operatorname{Pr} \sum_{r=0}^{m-1}\left(f_{m-1-r}^{\prime} \theta_{r}-c f_{m-1-r} \theta_{r}^{\prime}\right) .
\end{aligned}
$$

Here, $\chi_{m}$ is defined as

$$
\chi_{m}= \begin{cases}0, & \text { for } m \leq 1, \\ 1, & \text { for } m>1\end{cases}
$$

The $m$ th order $(m \geq 1)$ deformation equations (27) are linear, and using the initial guesses $f_{0}(\eta)$, $\theta_{0}(\eta)$, we can obtain $f_{1}(\eta), f_{2}(\eta), f_{3}(\eta)$, and so forth and $\theta_{1}(\eta), \theta_{2}(\eta), \theta_{3}(\eta)$, and so forth using boundary conditions (28). With the help of the symbolic computation 
software MATHEMATICA, the solution series for (27) and (28) can be expressed in the following form:

$$
\begin{aligned}
& f(\eta)=\sum_{m=0}^{\infty} f_{m}(\eta)=\lim _{M \rightarrow \infty}\left[\sum_{n=1}^{4} \sum_{m=n-1}^{M+34} a_{m, n} \eta^{n}\right], \\
& \theta(\eta)=\sum_{m=0}^{\infty} \theta_{m}(\eta)=\lim _{M \rightarrow \infty}\left[\sum_{n=1}^{4+1} \sum_{m=n-1}^{4 M} b_{m, n} \eta^{n}\right] .
\end{aligned}
$$

The coefficient $a_{m, n}$ and $b_{m, n}$ can be obtained using the following recurrence relations:

$$
\begin{gathered}
a_{m, 0}=0, \\
a_{m, 1}=\chi_{m} a_{m-1,1}+\frac{2 K}{4 K+\alpha} \sum_{k=4}^{4 m+3} \frac{\Gamma_{m, k-4}}{k(k-1)(k-2)}, \\
a_{m, 2}=\chi_{m} a_{m-1,2}+\frac{\alpha}{4 K+\alpha} \sum_{k=4}^{4 m+3} \frac{\Gamma_{m, k-4}}{k(k-1)(k-2)}, \\
a_{m, 3}=\chi_{m} a_{m-1,3} \\
-\sum_{k=4}^{4 m+3}\left[\left\{\frac{\alpha+2 K}{4 K+\alpha}(k-3)+1\right\} \frac{\Gamma_{m, k-4}}{k(k-1)(k-2)(k-3)}\right], \\
a_{m, k}=\chi_{m} a_{m-1, k}+\frac{\Gamma_{m, k-4}}{k(k-1)(k-2)(k-3)}, \\
4 \leq k \leq 4 m+3,
\end{gathered}
$$

where

$$
\begin{gathered}
\Gamma_{m, 1}^{k}=\hbar_{1}\left(a 4_{m-1, k}-\left(M^{2}\right) a 2_{m-1, k}\right. \\
\left.-R\left\{(2-c) \delta 1_{m, k}-c \delta 2_{m, k}\right\}\right), \quad 0 \leq k \leq 4 m-1, \\
\delta 1_{m, n}=\sum_{r=0}^{m-1} \sum_{k=\max \{0, n-4 m+4 r+1\}}^{\min \{n, 4 r+3\}} a 1_{r, k} a 2_{m-r-1, n-k}, \\
\delta 2_{m, n}=\sum_{r=0}^{m-1} \sum_{k=\max \{0, n-4 m+4 r+1\}}^{\min \{n, 4 r+3\}} \quad a_{r, k} a 3_{m-r-1, n-k}, \\
a 4_{m, k}=(k+1) a 3_{m, k+1}, \quad 0 \leq k \leq 4 m-1, \\
a 3_{m, k}=(k+1) a 2_{m, k+1}, \quad 0 \leq k \leq 4 m, \\
a 2_{m, k}=(k+1) a 1_{m, k+1}, \quad 0 \leq k \leq 4 m+1, \\
a 1_{m, k}=(k+1) a a_{m, k+1}, \quad 0 \leq k \leq 4 m+2,
\end{gathered}
$$

$$
\begin{gathered}
b_{m, 0}=0 \\
b_{m, 1}=\chi_{m} b_{m-1,1}-\sum_{k=2}^{4 m+1} \frac{\gamma_{m, k-2}}{k(k-1)}, \\
b_{m, k}=\chi_{m} b_{m-1, k}+\frac{\gamma_{m, k-2}}{k(k-1)}, \quad 2 \leq k \leq 4 m+1,
\end{gathered}
$$

where

$$
\begin{gathered}
\gamma_{m, 1}^{k}=\hbar_{2}\left(b 2_{m-1, k}-R \operatorname{Pr}\left\{\Delta 1_{m, k}-c \Delta 2_{m, k}\right\}\right), \\
0 \leq k \leq 4 m-1, \\
\Delta 1_{m, n}=\sum_{r=0}^{m-1} \sum_{k=\max \{0, n-4 m+4 r+1\}}^{\min \{n, 4 r+3\}} a 1_{r, k} b_{m-r-1, n-k}, \\
\Delta 2_{m, n}=\sum_{r=0}^{m-1} \sum_{k=\max \{0, n-4 m+4 r+1\}}^{\min \{n, 4 r+3\}} \quad a_{r, k} b 1_{m-r-1, n-k}, \\
b 2_{m, k}=(k+1) b 1_{m, k+1}, \quad 0 \leq k \leq 4 m-1, \\
b 1_{m, k}=(k+1) b_{m, k+1}, \quad 0 \leq k \leq 4 m .
\end{gathered}
$$

\section{Convergence of the HAM Solutions}

Equations (31) are the series solutions of the present problem of MHD flow and heat transfer in a horizontal channel bounded by a shrinking sheet and a porous bed of very small permeability. As Liao [40] pointed out that the convergence region and convergence rate of the HAM solution series can be adjusted by means of the value of the auxiliary parameters $\hbar_{1}$ and $\hbar_{2}$ here we have taken $\hbar_{1}=\hbar_{2}=\hbar$. In order to find the convergence region for the series (31), we have plotted the socalled h-curves. We have plotted the curves of $f^{\prime \prime}(0)$ versus $\hbar$ and $\theta^{\prime}(0)$ versus $\hbar$ in Figure 2, for 20th, 21st, and 22nd order approximation. It clearly indicates that the valid region of $\hbar$ is about $-1.8 \leq \hbar \leq-0.4$. In all numerical calculations for plotting graphs, the order of approximation is taken as $m=22$ and $\hbar=-1.1$.

\section{Discussion}

In the present investigation, the effects of a porous bed and a shrinking wall on the MHD flow and heat transfer are examined in a horizontal channel. The governing nonlinear partial differential equations for flow and heat transfer are converted into ordinary differential equations using similarity transformations. Then series solutions are obtained for velocity and temperature fields using homotopy analysis method (HAM). The convergence region of the solution series by HAM is obtained by plotting the so-called hcurve graph in Figure 2. To ensure the steady flow near the 


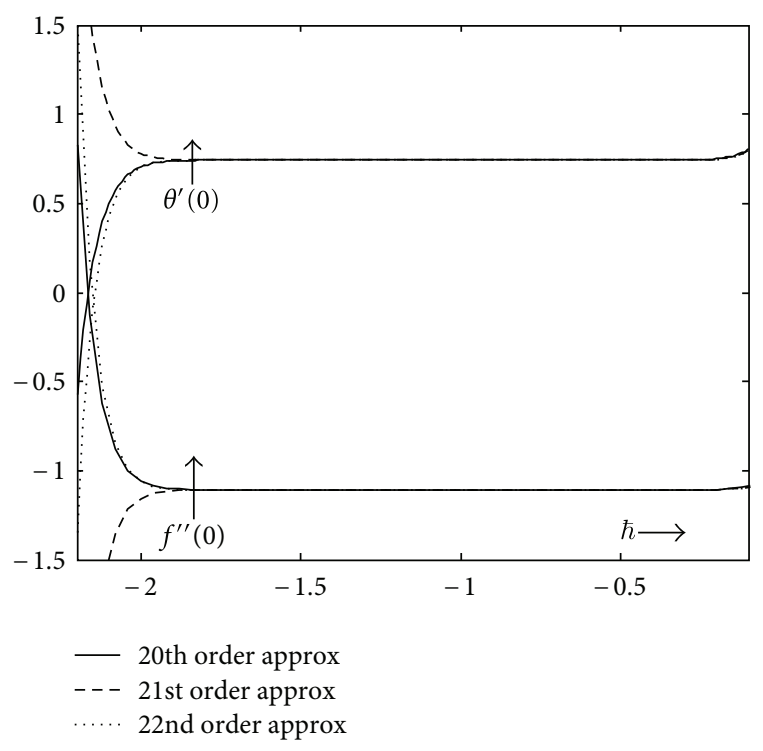

Figure 2: h-curve for $f^{\prime \prime}(0)$ and $\theta^{\prime}(0)$ for $M=1, c=3, K=0.001$, $\lambda=0.5, \alpha=0.1, \operatorname{Re}=2$, and $\operatorname{Pr}=2$.

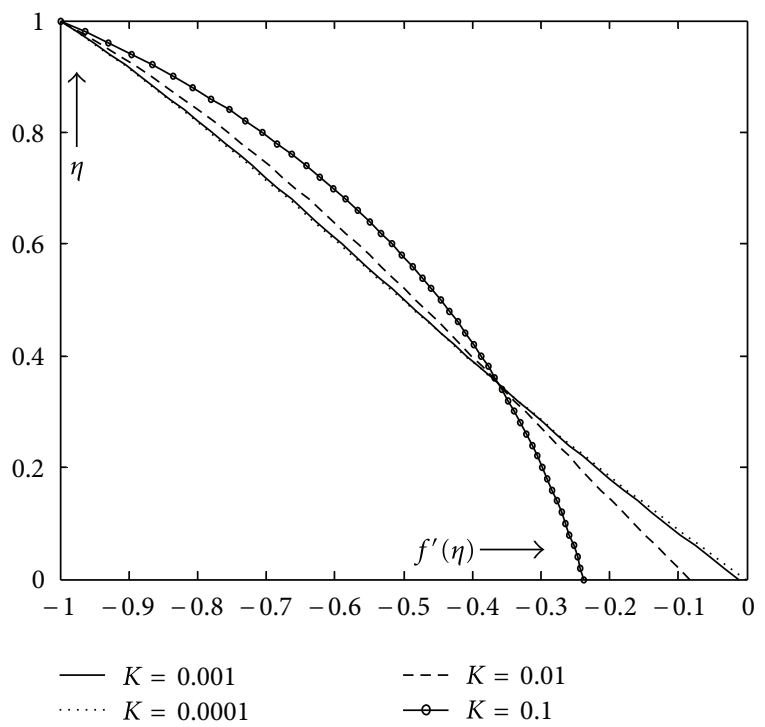

Figure 3: $f^{\prime}(\eta)$ for $M=1, c=3, \alpha=0.1, \lambda=0.5$, and $R=2$.

shrinking sheet by confining the generated vorticity in the boundary layer, the opposite forces such as the wall mass suction and the magnetic field are taken as sufficiently high by assigning suitable values of $M$ or $\lambda$.

Figures 3, 4, 5, 6, and 7 illustrate the variations of axial velocity for various values of the pertinent parameters. Figure 3 shows that magnitude of the axial velocity increases near the porous bed in the channel with the increase of the permeability parameter $(K)$, while it decreases near the upper shrinking sheet. Permeability is the measure of ease to flow and it enhances fluid flow near the porous bed. The effect of the fluid saturated porous medium bed is to introduce a slip at one wall of the channel, and therefore the flow velocity at this wall is no longer equal to zero. This slip

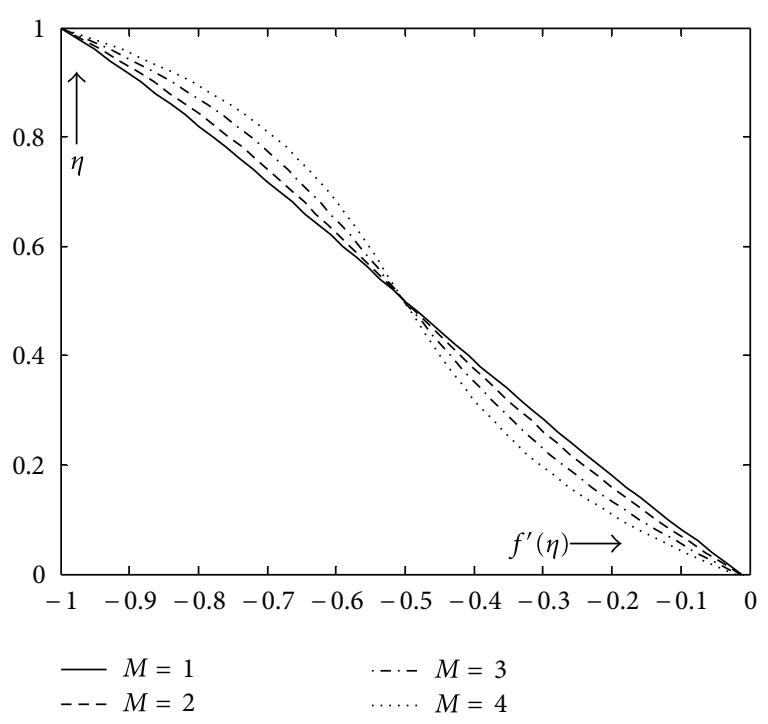

Figure 4: $f^{\prime}(\eta)$ for $c=3, K=0.001, \lambda=0.5, \alpha=0.1$, and $R=2$.

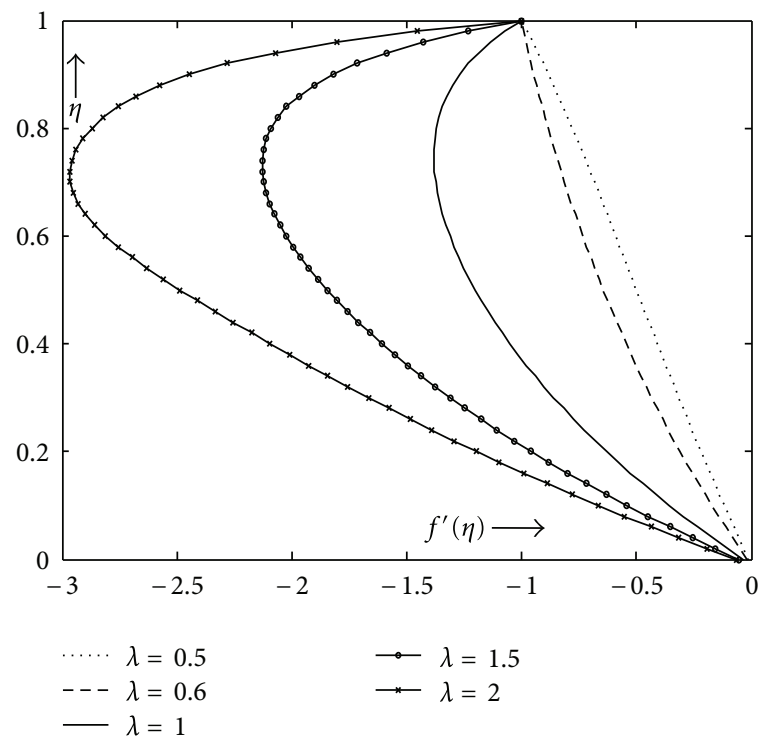

Figure 5: $f^{\prime}(\eta)$ for $M=1, c=3, K=0.001, \alpha=0.1$, and $R=2$.

depends on the parameters of structure and permeability of the porous matrix and affects the flow inside the channel through boundary condition suggested by Saffman [39]. In Figure 4, we observe that the magnitude of the axial velocity decreases with the increase of the magnetic parameter $(M)$ near the upper shrinking sheet since Lorentz force opposes the flow and leads to the deceleration of fluid flow near upper wall, while it increases near porous bed. Figure 5 illustrates that with increase in the value of suction parameter $(\lambda)$, magnitude of the axial flow increases in the channel. Figure 6 shows that the effect of the shrinking parameter $(R)$ is to reduce the flow near the upper sheet while the reverse effect is observed near the lower porous bed. Figure 7 depicts that the axial flow near the upper wall is greater when the sheet shrinks axisymmetrically than that when it shrinks only in 


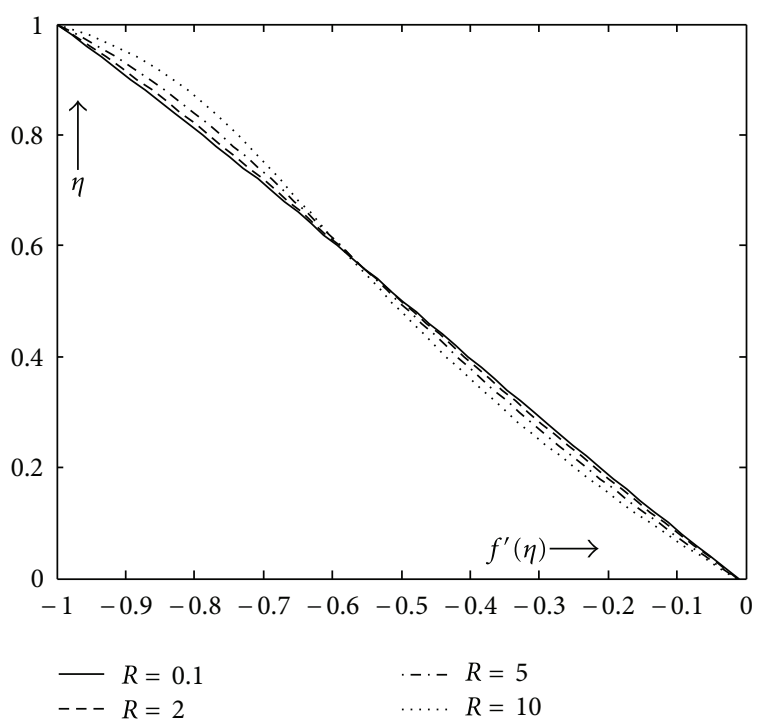

Figure 6: $f^{\prime}(\eta)$ for $M=1, c=3, K=0.001, \lambda=0.5$, and $\alpha=0.1$.

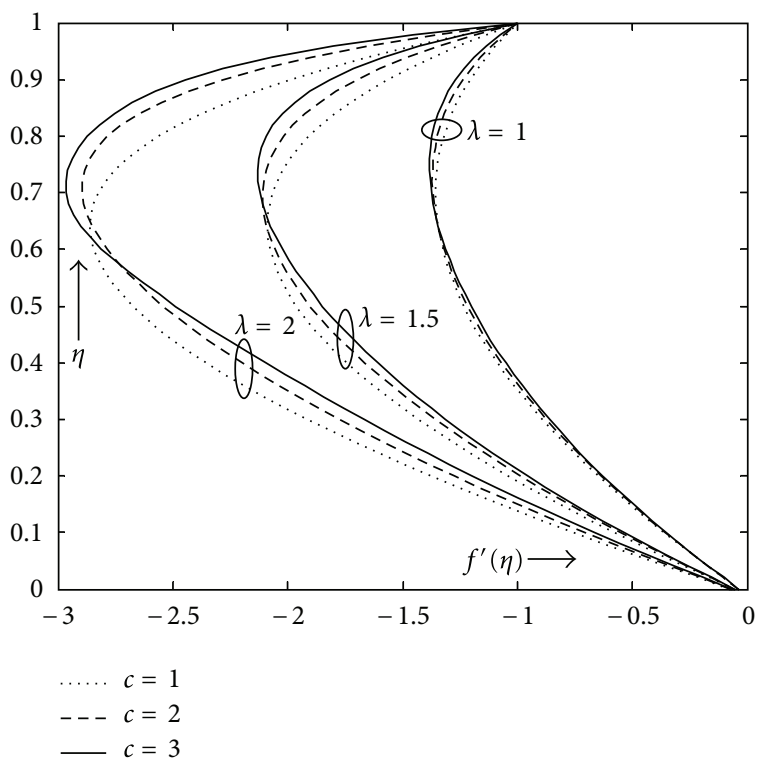

Figure 7: $f^{\prime}(\eta)$ for $M=1, K=0.001, \alpha=0.1$, and $R=2$.

the $x$ direction, while near the porous bed reverse effect is observed.

Figures $8,9,10,11,12$, and 13 illustrate profiles of the nondimensional temperature against the coordinate $\eta$. The effect of Prandtl number (Pr) on the temperature profiles can be seen in Figure 8. Increase in the Pr values leads to the decrease of the temperature in the channel and the thickness of the thermal boundary layer becomes smaller. As Pr value increases, the thermal diffusivity decreases which reduces the energy transfer ability; hence the temperature decreases. Note that very small values of $\operatorname{Pr}$ correspond to liquid metals, $\operatorname{Pr} \sim 1$ corresponds to air and diatomic gases, and very large values of Pr correspond to highly viscous oils. At room temperature, $\operatorname{Pr} \sim 7$ corresponds to water. Figure 9 depicts

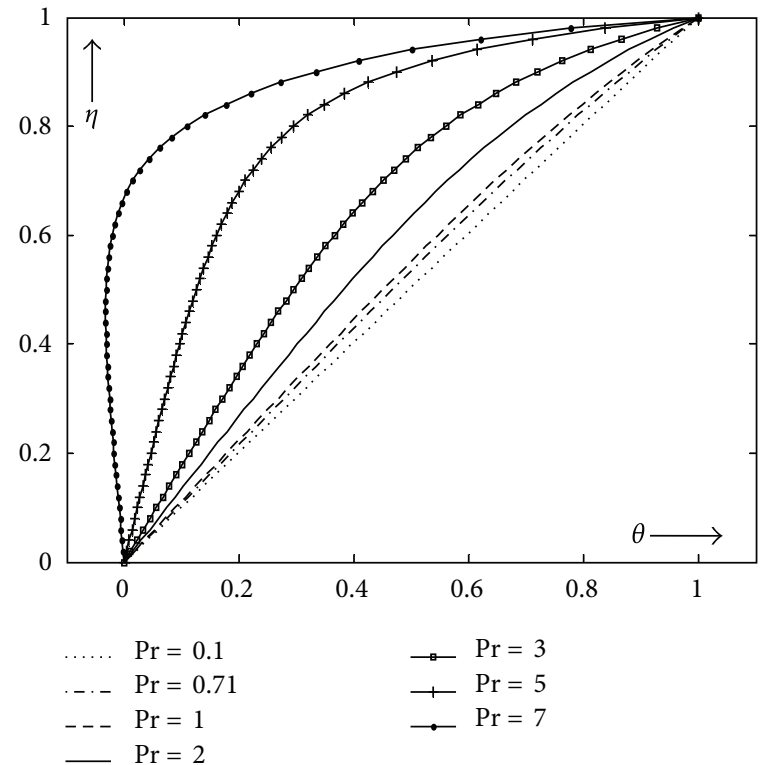

Figure 8: $\theta(\eta)$ for $M=1, c=3, K=0.001, \lambda=0.5, \alpha=0.1$, and $R=$ 2 .

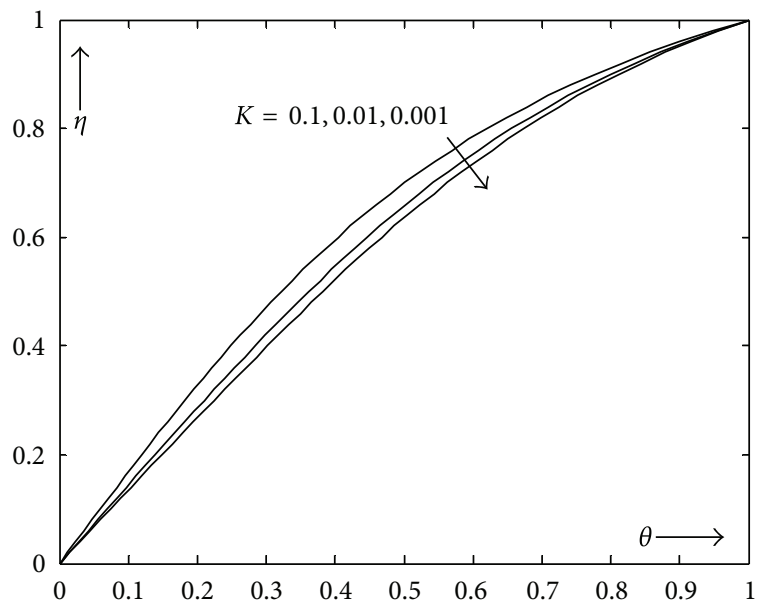

Figure 9: $\theta(\eta)$ for $M=1, c=3, \lambda=0.5, \alpha=0.1, R=2$, and $\operatorname{Pr}=2$.

temperature distribution in the channel for various values of the permeability parameter $(K)$. It is found that the effect of the permeability of lower bed is to decrease the temperature throughout the channel. It is also observed in Figures 10, 11, and 12 that the temperature in the channel decreases with increase in the value of suction parameter $(\lambda)$ or magnetic parameter $(M)$ or shrinking parameter $(R)$. Further, it is observed in Figure 13 that the temperature throughout the channel for the axisymmetric shrinking case $(c=2)$ is less than that for the case of one-direction shrinking $(c=1)$.

Many industrial processes, for example, in metallurgical and packaging units, involve the drawing and cooling of continuous strips, sheets, and so forth and there quality depends on the rate of cooling and shear stresses on them. Therefore the skin friction coefficient and the rate of heat transfer are the physical quantities of interest to engineers 


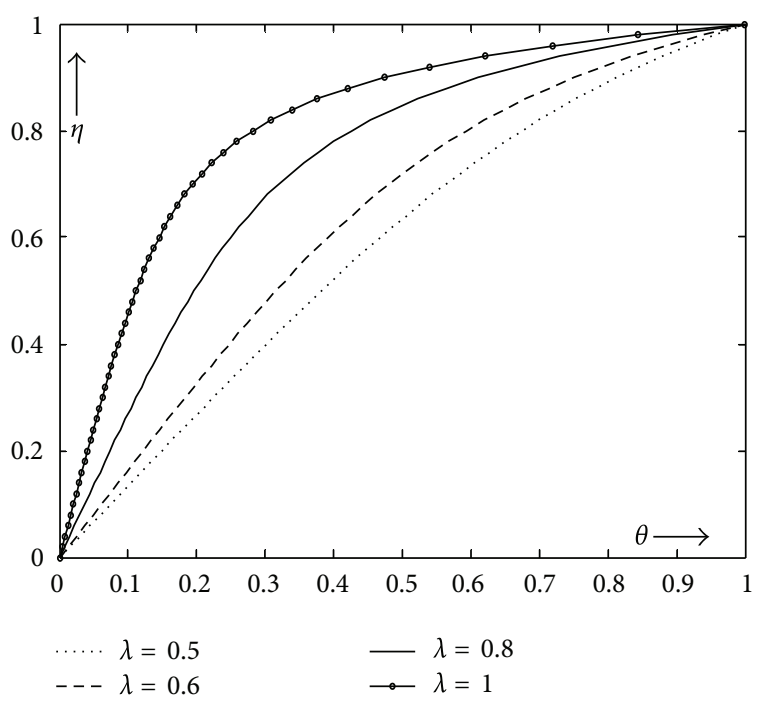

Figure 10: $\theta(\eta)$ for $M=1, c=3, K=0.001, R=2, \operatorname{Pr}=2$, and $\alpha=$ 0.1 .

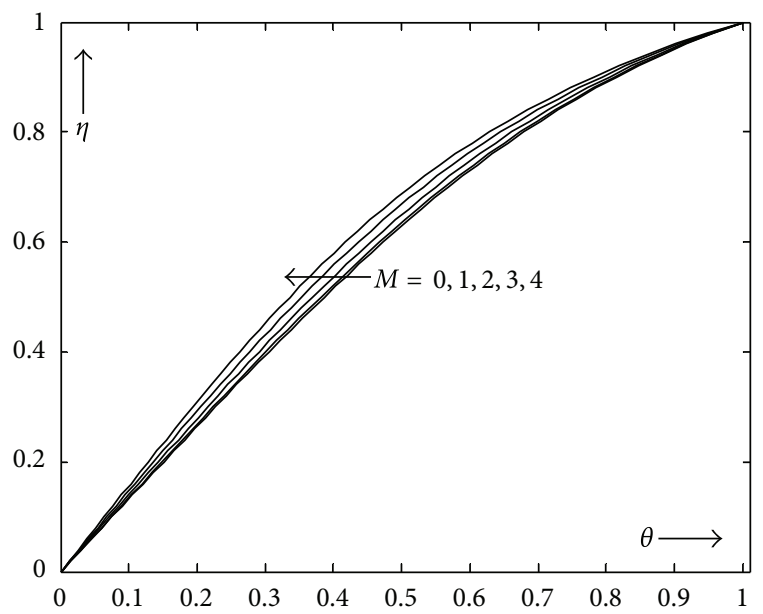

Figure 11: $\theta(\eta)$ for $\operatorname{Pr}=2, c=3, K=0.001, \lambda=0.5, \alpha=0.1$, and $R=$ 2.

and these are plotted in Figures 14 and 15 for various values of the pertinent parameters. It is found that the magnetic field increases the surface shear stress for axial flow, that is, the local skin friction at the shrinking sheet $\eta=1$, because an increase in the strength of magnetic field leads to a thinner boundary layer, thereby causing an increase in the velocity gradient $f^{\prime \prime}(1)$ at the sheet. Shrinking parameter $(R)$ or permeability parameter $(K)$ also leads to an increase in the skin friction at the shrinking sheet, whereas the effect of the suction parameter $(\lambda)$ is to reduce skin friction at the shrinking sheet. It is also noticed that the skin friction at shrinking sheet for axisymmetric shrinking is greater than that for the case of one-direction shrinking. It is observed in Figure 15 that the temperature gradient at the shrinking sheet increases as Prandtl number $(\mathrm{Pr})$ increases, since a higher $\mathrm{Pr}$ fluid is more viscous and has a thinner thermal boundary layer which results at the sheet in a higher rate of heat

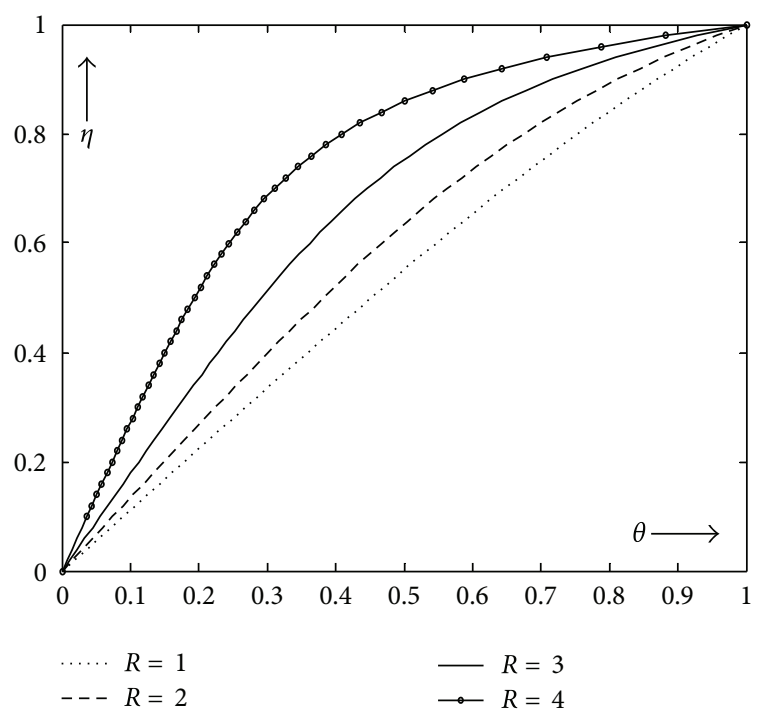

Figure 12: $\theta(\eta)$ for $M=1, c=3, \lambda=0.5, \alpha=0.1, K=0.001$, and $\operatorname{Pr}=2$.

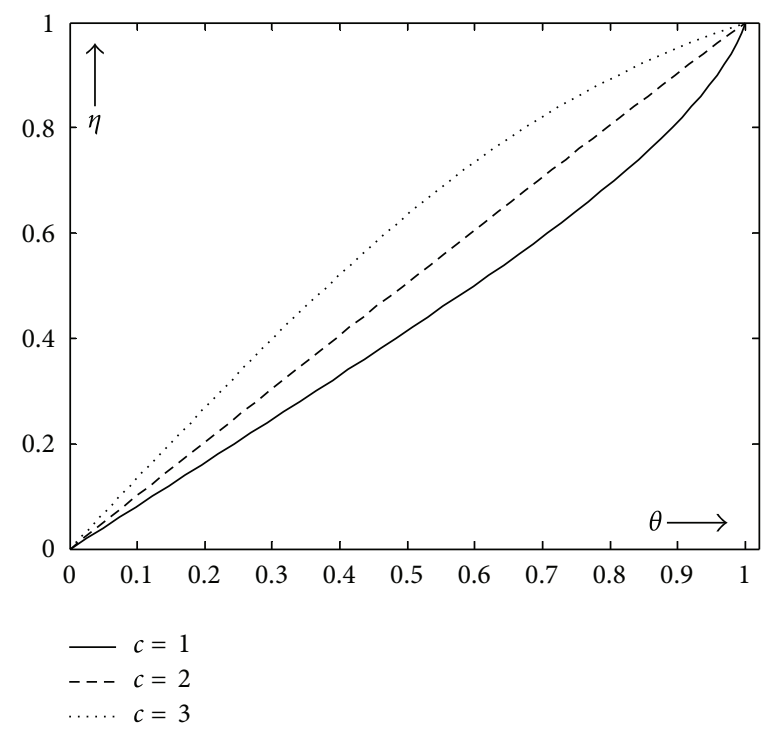

Figure 13: $\theta(\eta)$ for $M=1, \lambda=0.5, K=0.001, R=2, \operatorname{Pr}=2$, and $\alpha=$ 0.1 .

transfer. It is also noticed that the temperature gradient at the shrinking sheet increases with the increase in the value of the shrinking parameter $(R)$, or permeability $(K)$, or suction parameter $(\lambda)$, or magnetic parameter $(M)$. It is also observed that the temperature gradient at the shrinking sheet is greater for the axisymmetric shrinking case than to one-direction shrinking.

\section{Conclusion}

The present paper studies the flow and heat transfer in a channel bounded by a porous medium bed and a shrinking sheet in the presence of a magnetic field. Using similarity 


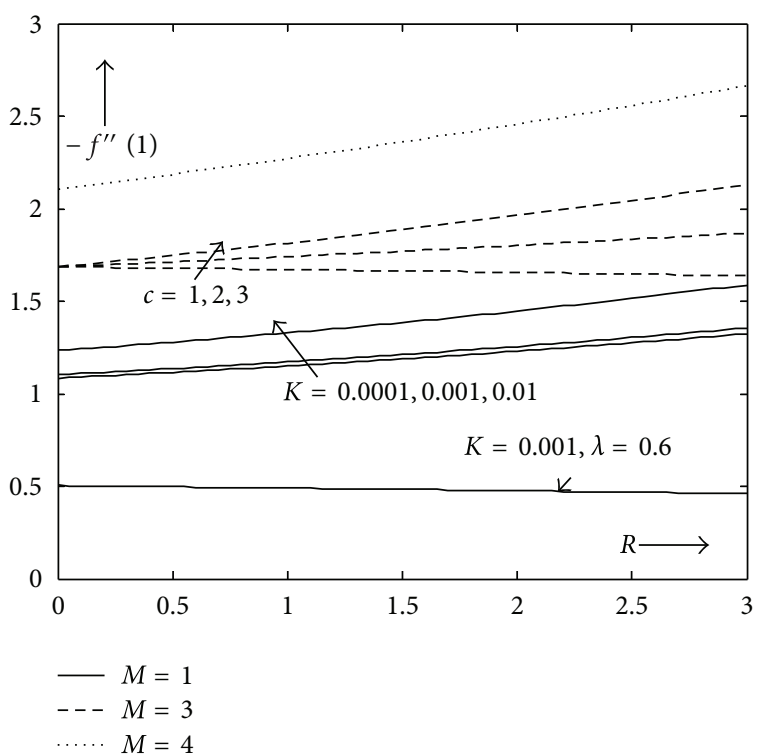

FIgURE 14: $-f^{\prime \prime}(1)$ versus $R$ for $c=3, K=0.001, \lambda=0.5$, and $\alpha=0.1$.

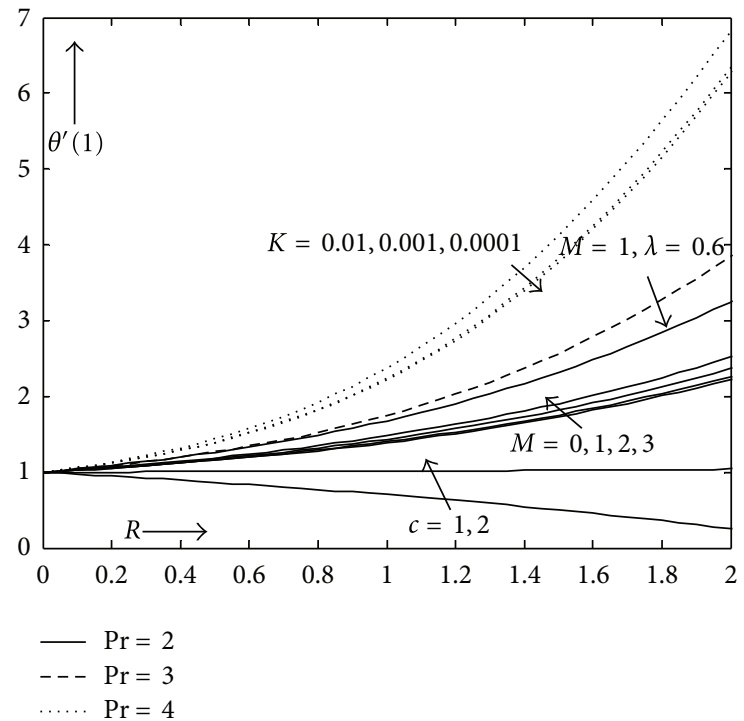

Figure 15: $\theta^{\prime}(1)$ versus $R$ for $M=1, c=3, K=0.001, \lambda=0.5$, and $\alpha$ $=0.1$.

transformations and homotopy analysis method, analytical series solutions are obtained and presented graphically to discuss features of these solutions for various values of the pertinent parameters. Convergence of the HAM series solution is ensured by taking suitable value of the parameter $h$. It is observed that the axial flow in the channel adjacent to upper wall is greater when it shrinks axisymmetrically to that when it shrinks in one direction, while reverse effect is observed at the other wall. Further, the effect of suction parameter is to increase the axial flow in the channel. Temperature in the channel decreases as the value of Prandtl number increases, and the effect of permeability parameter of the lower porous bed is to decrease temperature throughout the channel. It is also found that the temperature in the channel for the axisymmetric shrinking case is less than that for the case of one-direction shrinking. It is seen that the physical parameters such as suction and magnetic field control the rate of cooling and skin friction at the shrinking surface.

\section{Acknowledgment}

The support provided by the Council of Scientific and Industrial Research through Senior Research Fellowship to one of the authors, Rashmi Agrawal, is gratefully acknowledged.

\section{References}

[1] L. J. Crane, "Flow past a stretching plate," Zeitschrift für Angewandte Mathematik und Physik, vol. 21, no. 4, pp. 645-647, 1970.

[2] K. R. Rajagopal, T. Y. Na, and A. S. Gupta, "Flow of a viscoelastic fluid over a stretching sheet," Rheologica Acta, vol. 23, no. 2, pp. 213-215, 1984.

[3] B. S. Dandapat and A. S. Gupta, "Flow and heat transfer in a viscoelastic fluid over a stretching sheet," International Journal of Non-Linear Mechanics, vol. 24, no. 3, pp. 215-219, 1989.

[4] T. C. Chiam, "Hydromagnetic flow over a surface stretching with a power-law velocity," International Journal of Engineering Science, vol. 33, no. 3, pp. 429-435, 1995.

[5] R. Cortell, "Flow and heat transfer of a fluid through a porous medium over a stretching surface with internal heat generation/absorption and suction/blowing," Fluid Dynamics Research, vol. 37, no. 4, pp. 231-245, 2005.

[6] I. C. Liu, "Flow and heat transfer of an electrically conducting fluid of second grade in a porous medium over a stretching sheet subject to a transverse magnetic field," International Journal of Non-Linear Mechanics, vol. 40, no. 4, pp. 465-474, 2005.

[7] P. D. Ariel, T. Hayat, and S. Asghar, "The flow of an elasticoviscous fluid past a stretching sheet with partial slip," Acta Mechanica, vol. 187, no. 1-4, pp. 29-35, 2006.

[8] P. D. Ariel, T. Hayat, and S. Asghar, "Homotopy perturbation method and axisymmetric flow over a stretching sheet," International Journal of Nonlinear Sciences and Numerical Simulation, vol. 7, no. 4, pp. 399-406, 2006.

[9] Z. Abbas, Y. Wang, T. Hayat, and M. Oberlack, "Hydromagnetic flow in a viscoelastic fluid due to the oscillatory stretching surface," International Journal of Non-Linear Mechanics, vol. 43, no. 8, pp. 783-793, 2008.

[10] M. Abd El-Aziz, "Flow and heat transfer over an unsteady stretching surface with Hall effect," Meccanica, vol. 45, no. 1, pp. 97-109, 2010.

[11] D. S. Chauhan and P. Rastogi, "Heat transfer and entropy generation in MHD flow through porous medium past a stretching sheet," International Journal of Energy and Technology, vol. 3, pp. $1-13,2011$.

[12] D. S. Chauhan and R. Agrawal, "MHD flow through a porous medium adjacent to a stretching sheet: numerical and an approximate solution," European Physical Journal Plus, vol. 126, article 47, 2011.

[13] D. S. Chauhan and A. Olkha, "Slip flow and heat transfer of a second-grade fluid in a porous medium over a stretching sheet with power-law surface temperature or heat flux," Chemical 
Engineering Communications, vol. 198, no. 9, pp. 1129-1145, 2011.

[14] C. Y. Wang, "Liquid film on an unsteady stretching sheet," Quarterly of Applied Mathematics, vol. 48, pp. 601-610, 1990.

[15] M. Miklavčič and C. Y. Wang, "Viscous flow due to a shrinking sheet," Quarterly of Applied Mathematics, vol. 64, no. 2, pp. 283-290, 2006.

[16] C. Y. Wang, "Stagnation flow towards a shrinking sheet," International Journal of Non-Linear Mechanics, vol. 43, no. 5, pp. 377-382, 2008.

[17] S. Nadeem and M. Awais, "Thin film flow of an unsteady shrinking sheet through porous medium with variable viscosity," Physics Letters A, vol. 372, no. 30, pp. 4965-4972, 2008.

[18] T. Fang, W. Liang, and C. F. F. Lee, "A new solution branch for the Blasius equation-A shrinking sheet problem," Computers and Mathematics with Applications, vol. 56, no. 12, pp. 3088-3095, 2008.

[19] Muhaimin, R. Kandasamy, and A. B. Khamis, "Effects of heat and mass transfer on nonlinear MHD boundary layer flow over a shrinking sheet in the presence of suction," Applied Mathematics and Mechanics, vol. 29, no. 10, pp. 1309-1317, 2008.

[20] M. Rahimpour, C. R. Mohebpour, A. Kimiaeifar, and G. H. Bagheri, "On the analytical solution of axisymmetric stagnation flow towards a shrinking sheet," International Journal of Mechanical, vol. 2, pp. 1-10, 2008.

[21] T. Hayat, T. Javed, and M. Sajid, "Analytic solution for MHD rotating flow of a second grade fluid over a shrinking surface," Physics Letters A, vol. 372, no. 18, pp. 3264-3273, 2008.

[22] M. Sajid and T. Hayat, "The application of homotopy analysis method for MHD viscous flow due to a shrinking sheet," Chaos, Solitons and Fractals, vol. 39, no. 3, pp. 1317-1323, 2009.

[23] T. Fang, "Boundary layer flow over a shrinking sheet with power-law velocity," International Journal of Heat and Mass Transfer, vol. 51, no. 25-26, pp. 5838-5843, 2008.

[24] T. Fang and J. Zhang, "Closed-form exact solutions of MHD viscous flow over a shrinking sheet," Communications in Nonlinear Science and Numerical Simulation, vol. 14, no. 7, pp. 2853-2857, 2009.

[25] T. Fang, J. Zhang, and S. Yao, "Viscous flow over an unsteady shrinking sheet with mass transfer," Chinese Physics Letters, vol. 26, Article ID 014703, 2009.

[26] B. Yao and J. Chen, "A new analytical solution branch for the Blasius equation with a shrinking sheet," Applied Mathematics and Computation, vol. 215, no. 3, pp. 1146-1153, 2009.

[27] N. F. Mohd and I. Hashim, "MHD flow and heat transfer adjacent to a permeable shrinking sheet embedded in a porous medium," Sains Malaysiana, vol. 38, no. 4, pp. 559-565, 2009.

[28] R. Cortell, "On a certain boundary value problem arising in shrinking sheet flows," Applied Mathematics and Computation, vol. 217, no. 8, pp. 4086-4093, 2010.

[29] N. F. M. Noor, S. Awang Kechil, and I. Hashim, "Simple nonperturbative solution for MHD viscous flow due to a shrinking sheet," Communications in Nonlinear Science and Numerical Simulation, vol. 15, no. 2, pp. 144-148, 2010.

[30] T. Hayat, S. Iram, T. Javed, and S. Asghar, "Shrinking flow of second grade fluid in a rotating frame: an analytic solution," Communications in Nonlinear Science and Numerical Simulation, vol. 15, no. 10, pp. 2932-2941, 2010.

[31] T. Fang and J. Zhang, "Thermal boundary layers over a shrinking sheet: an analytical solution," Acta Mechanica, vol. 209, no. 3-4, pp. 325-343, 2010.
[32] T. Fang, S. Yao, J. Zhang, and A. Aziz, "Viscous flow over a shrinking sheet with a second order slip flow model," Communications in Nonlinear Science and Numerical Simulation, vol. 15, no. 7, pp. 1831-1842, 2010.

[33] A. Ishak, Y. Y. Lok, and I. Pop, "Stagnation-point flow over a shrinking sheet in a micropolar fluid," Chemical Engineering Communications, vol. 197, no. 11, pp. 1417-1427, 2010.

[34] J. F. Brady and A. Acrivos, "Steady flow in a channel or tube with an accelerating surface velocity. An exact solution to the NavierStokes equations with reverse flow," Journal of Fluid Mechanics, vol. 112, pp. 127-150, 1981.

[35] A. K. Borkakoti and A. Bharali, "Hydromagnetic flow and heat transfer between two horizontal plates, the lower plate being a stretching sheet," Quarterly of Applied Mathematics, vol. 40, no. 4, pp. 461-467, 1983.

[36] D. S. Chauhan and P. K. Jakhar, "Non-Newtonian stretching coupled-flow in a channel bounded by a highly porous layer," Modelling, Measurement and Control B, vol. 71, no. 3-4, pp. 33-40, 2002.

[37] T. Hayat, Z. Abbas, T. Javed, and M. Sajid, "Three-dimensional rotating flow induced by a shrinking sheet for suction," Chaos, Solitons and Fractals, vol. 39, no. 4, pp. 1615-1626, 2009.

[38] D. S. Chauhan and R. Agrawal, "MHD flow and heat transfer in a channel bounded by a shrinking sheet and a plate with porous substrate," Journal of Engineering Physics and Thermophysics, vol. 84, pp. 1034-1046, 2011.

[39] P. G. Saffman, "On the boundary condition at the surface of a porous medium," Studies in Applied Mathematics, vol. 50, no. 2, pp. 93-101, 1971.

[40] S. J. Liao, Beyond Perturbation: Introduction to the Homotopy Analysis Method, Chapman and Hall, Boca Raton, Fla, USA, 2004. 

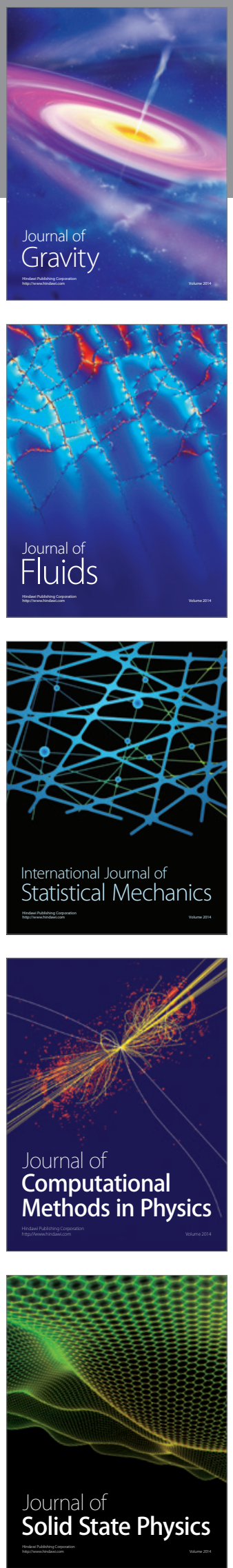

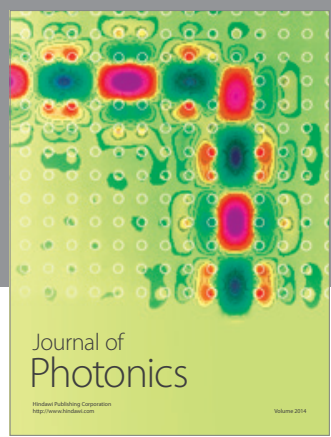

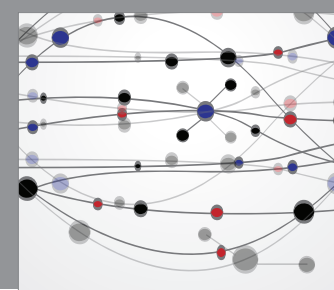

The Scientific World Journal

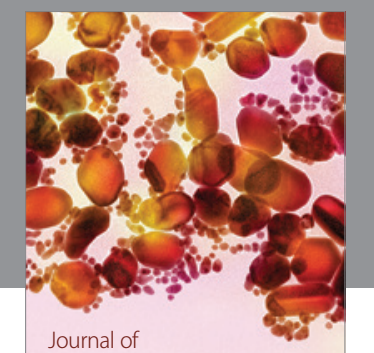

Soft Matter
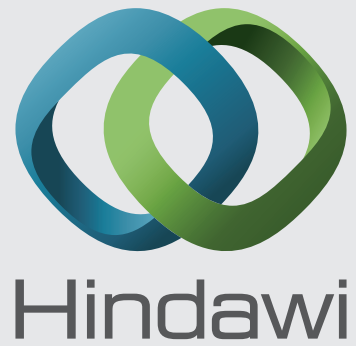

Submit your manuscripts at

http://www.hindawi.com
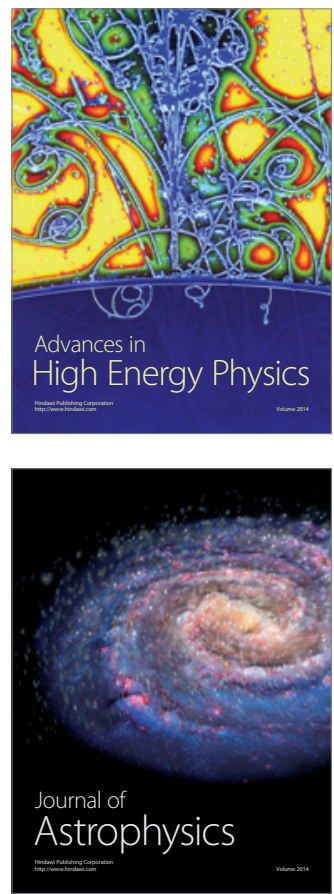
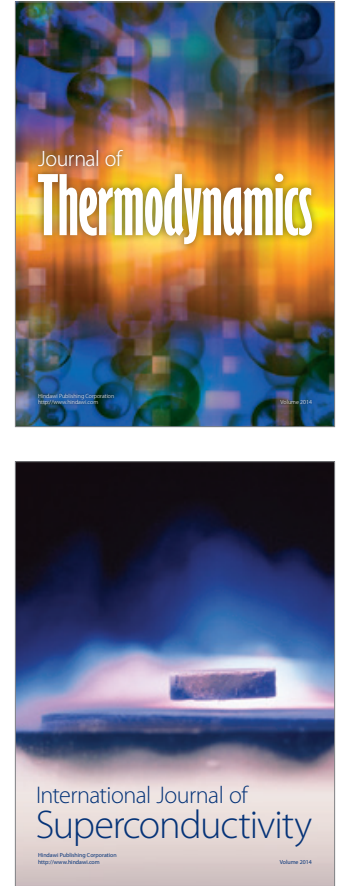
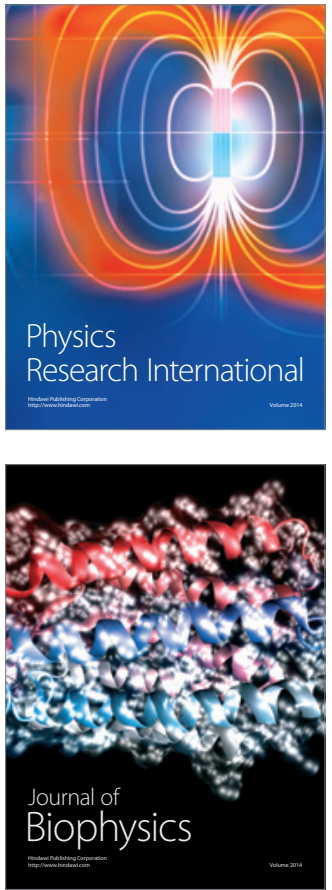
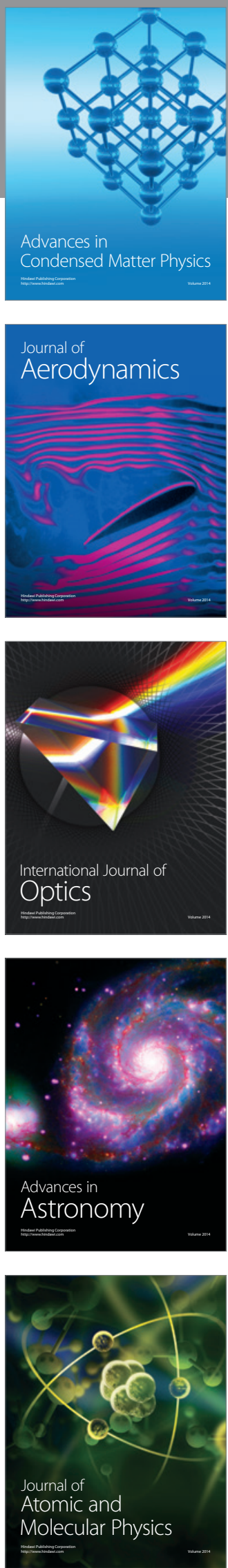